\title{
Energy Dependence of Parameters Characterizing Multiply Backscattering of Gamma Photons
}

\author{
Arvind D Sabharwal, Bhajan Singh \& B.S. Sandhu* \\ Physics Department, Punjabi University, Patiala-147002, India \\ *E-mail: balvir@pbi.ac.in; balvirss99@yahoo.com
}

\begin{abstract}
The present studies aimed to investigate the effects of energy dependence of parameters characterizing multiply backscattering of gamma photons. The numbers of multiply backscattered events are found to be increasing with thickness of copper target, and saturate for a particular thickness known as saturation thickness. The saturation thickness is found to be decreasing with increase in incident gamma photon energy, and also is not altered by the variation in collimator opening. The number, energy and dose albedos, characterizing the reflection probability of a material, are also evaluated. For each of the incident gamma photon energy, the number and energy albedos show an increase with increasing target thickness, and finally saturate. Monte Carlo calculations support the results of present experimental work.
\end{abstract}

Keywords: Multiply backscattering, Saturation depth, Number, energy and dose albedos, Monte Carlo simulations

\section{INTRODUCTION}

The scattering of gamma photons backward from the bulk of a material is named as backscattering of gamma rays. The backscattering (or reflection) of gamma photons is of fundamental importance in Compton profile, radiation shielding, industrial and medical applications. The quantities that characterize the reflection probability are called albedos or reflection factors. The nature of reflection of gamma photons incident on the surface of a scattering medium depends on the energy of the primary gamma flux. In literature the workers concern on the reflection characteristics of the material, such as total albedo, differential albedo, reflection build-up factors, exposure rate field distribution outside and on the scatterer. Hayward and Hubbel [1] and Pozdneyev [2] have provided useful information about intensity and energy distributions of gamma rays backscattered from various materials as a function of primary gamma radiation energy and geometry. Bulatov and Garusov [3] used a small dosimeter without a collimator and calculated the energy albedos for gamma photons from ${ }^{60} \mathrm{Co}$ and ${ }^{198} \mathrm{Au}$. Hyodo [4] used a small point isotropic source on the surface of a scatterer and a $\mathrm{NaI}(\mathrm{Tl})$ scintillator without a collimator and calculated the number, energy albedo and energy distributions of backscattered gamma photons from semi infinite slabs of paraffin, aluminium, iron, tin and lead. Hayward and Hubbell [5] developed a Monte Carlo method to determine the albedo factor at $1 \mathrm{MeV}$ photons scattered from semiinfinite slabs of water, aluminium, copper, tin and lead at various angles of incidence.

\footnotetext{
Journal of Nuclear Physics, Material Sciences, Radiation and Applications Vol. 1, No. 2

February 2014 pp. 239-246
}

\section{CHITKARA 司 UNIVERSITY \\ (C2014 by Chitkara University. All Rights} Reserved. 
Sabharwal, A.D. Singh, B.

Sandhu, B.S.

Our previous measurements [6-7] and references therein, provide in brief the results of various experiments on multiple backscattering along with analytical and Monte Carlo simulation approaches to study these processes. These measurements have confirmed that the saturation thickness for multiply backscattered events decreases with increase in atomic number $(Z)$, and the detector response unfolding converting the observed pulse-height distributions to a true photon energy spectrum is quite satisfactory.

The multiply backscattered photons continue to decrease in energy as the number of scatterings increases in the target. Theses gamma photons of lower energy get registered in the spectrum along with the singly scattered events. The energy spectrum of such photons is broad and never completely separate from the singly scattered distribution. This makes rather difficult to judge the exact contribution of multiply scattered photons in the lower energy region near the backscattered peak. In present experiments, the problem is to quantitatively calculate the amount of multiply gamma photons backscattered from the finite slabs of copper, where the reflected component is mainly determined by multiple and not single scattering of gamma photon in the given target of finite thickness in accordance with the incident gamma photon energy. The present measurements also provide energy dependence of various parameters characterizing multiply backscattering of gamma photons.

\section{EXPERIMENTAL SET-UP AND METHOD OF MEASUREMENTS}

For accurate measurements of multiple backscattering and albedo factors, narrow or broad beam geometry can be used. Broad beam geometry, removing collimator in Fig. 1, allows significant number of backscattered gamma photons to enter into the detector where as in narrow beam geometry, the numbers of backscattered gamma photons entering in detector face are effectively reduced due to detector collimation. For the present measurements 279-, 320-, 511-, and $662 \mathrm{keV}$ gamma rays are obtained from the respective radioactive sources of ${ }^{203} \mathrm{Hg},{ }^{51} \mathrm{Cr},{ }^{22} \mathrm{Na}$, and ${ }^{137} \mathrm{Cs}$ respectively, having strength of the order of micro-Curie $\left(1 \mathrm{Ci}=3.7 \times 10^{10} \mathrm{~Bq}\right)$. Each of the radioactive source is placed adjacent to the center of the surface (facing toward detector) of copper target The beam of gamma rays from the radioactive source impinges on the rectangular targets of copper having size $8 \times 4 \mathrm{~cm}^{2}$. A NaI(Tl) gamma detector, 51 $\mathrm{mm}$ diameter and $51 \mathrm{~mm}$ thick, is placed at $180^{\circ}$ to the incident beam and the axes of radioactive source assembly and $\mathrm{NaI}(\mathrm{Tl})$ detector pass through centre of the target. The distance between the target and the detector is kept $120 \mathrm{~mm}$. The experimental data are accumulated on a PC based ORTEC Mastreo-32 Multi channel analyzer. The following sequence is adopted for present measurements of multiple backscattering of gamma rays:

(i) The gamma ray spectrum is recorded by placing the copper target, of known thickness, in the primary beam for a period of $10 \mathrm{ks}$. The spectrum (observed 


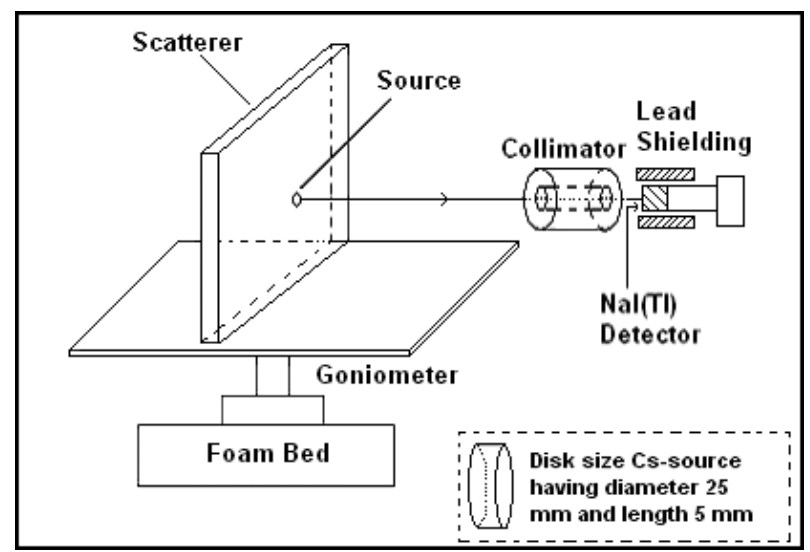

Energy Dependence of Parameters Characterizing Multiply Backscattering of Gamma Photons

Figure 1: Experimental set-up of present measurements

pulse-height distribution) accounts for incident (from source) and backscattered (from target) events.

(ii) The gamma ray spectrum is also recorded, after removing the target out of primary beam, for same duration of time to permit the registration of events due to photon flux from the source, cosmic rays and to any other process independent of target.

The subtraction of events under condition (ii) from those observed under condition (i) accounts for events originating from interactions of the primary gamma beam photons with target material only. The events under the observed backscattered peak of such a target related spectrum are composite of singly as well as multiply backscattered events.

Fig. 2 shows a typical observed pulse-height distribution (curve-a) with target of copper at scattering angle of $180^{\circ}$. Curve-b of Fig. 2 gives spectrum with the target out of the primary gamma beam. The subtraction of events under curve-b from those under curve-a results in scattered spectrum (curve-c) corresponding to events originating from the interaction of primary gamma photons with the target material and subsequent events such as multiple Compton scattering, bremsstrahlung, Rayleigh scattering etc. The curve-d is the analytically reconstructed singly backscattered distribution [6] originating from the copper target of known thickness. The experimental pulse-height distribution (curve-c) is converted to a photon energy spectrum with the help of an experimentally constructed 16 x 16 inverse response matrix [6]. This operation shifts the low pulse-height counts, resulting from partial absorption of higher energy photons, which are registered in the Compton continuum (in the lower bin meshes) to the energy region of the backscattered peak. The solid curve-e is the resulting calculated histogram of $\mathrm{N}(\mathrm{E})$ in units of photons, also known as response function of the scintillation detector. The response function of gamma 
Sabharwal, A.D. Singh, B.

Sandhu, B.S.
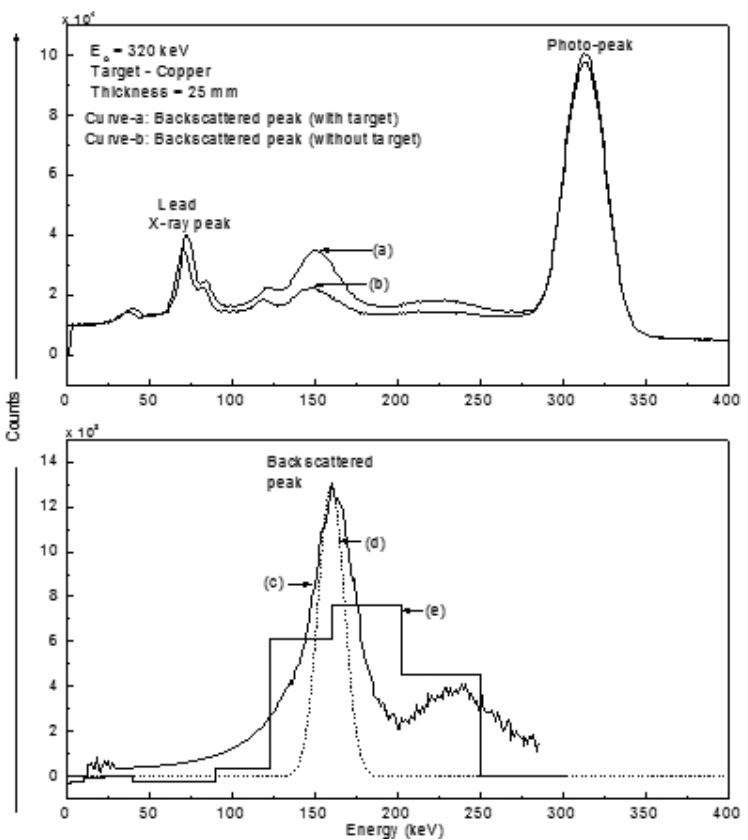

Figure 2: A typically observed pulse-height distribution with (curve-a) and without (curve-b) copper target in the primary $320 \mathrm{keV}$ gamma beam recorded for time duration of $10 \mathrm{ks}$. Experimentally observed pulse-height distribution (curve-c) obtained after subtracting background events (unrelated to target). Normalized analytically reconstructed singly backscattered full energy peak (curve-d) and resulting calculated histogram (curve-e) of converting pulse-height distribution to a true photon spectrum.

detector results in unfolding of observed gamma spectra and thus low pulse-height counts resulting from partial absorption of higher energy photons incident on the gamma detector are shifted to the photo-peak energy region. The events under the histogram in the Compton continuum accounts for photons of reduced energy (less than that of inelastically Compton backscattered peak) originating from multiple interactions in the target, bremsstrahlung and Rayleigh scattering, and finally escaping in the direction of gamma detector. The events under the calculated histogram corresponding to end points of backscattered energy peak accounts for singly and multiply scattered radiations (having energy equal to that of singly scattered ones). The events under curve-d of Fig. 2 are divided by peak-to-total ratio of the gamma detector and their subtraction from the events under the calculated histogram (curve-e) in the specified energy range results in events originating from 
Table 1: Experimentally measured values of saturation thickness (depth) in copper for different incident photon energies

\begin{tabular}{ccccc}
\hline \multirow{2}{*}{$\begin{array}{c}\text { Energy } \\
(\mathrm{keV})\end{array}$} & $\begin{array}{c}\text { Backscattered photon } \\
\text { energy }(\mathrm{keV})\end{array}$ & $\begin{array}{c}1 \text { m.f.p } \\
(\mathrm{mm})\end{array}$ & \multicolumn{2}{c}{ Measured saturation thickness } \\
\cline { 5 - 5 } 279 & 133.3 & 9.40 & in mm & in mean free path \\
320 & 142.1 & 10.40 & 17.5 & 1.86 \\
511 & 170.3 & 13.40 & 16.0 & 1.63 \\
662 & 184.3 & 15.30 & 15.5 & 1.19 \\
\hline
\end{tabular}

Energy Dependence of Parameters Characterizing Multiply Backscattering of Gamma Photons

multiple backscattering, having the same energy as in singly scattered distribution. These events, when corrected for the intrinsic (crystal) efficiency of the scintillation crystal, iodine escape peak and absorption in the Al-window of the detector, provide the emergent flux from the target under study at $180^{\circ}$ having energy in the range of inelastically backscattered peak. This procedure is then repeated for different thicknesses of copper target and detector collimator openings for various incident gamma photon energies.

\section{RESULTS AND DISCUSSION}

The plots of observed numbers of multiply backscattered events (having energy equal to singly backscattered ones) for different incident photon energies as a function of target thickness is shown in Fig. 3. The curves indicate that for each of the incident photon energy, the numbers of multiply backscattered events increases with increase in target thickness and then saturate after a particular value of target thickness, called saturation thickness (depth). The saturation of multiply scattered photons is due to the fact that as the thickness of target increases, the number of scattered events also increases but on the other hand an enhanced self-absorption results in decrease of the number of photons coming out of the target. So a stage is reached when the thickness of the target becomes sufficient to compensate the above increase and decrease of the number of photons. Saturation thickness for multiply backscattering of gamma ray photons is found to be decreasing with increase in incident gamma photons energy. This is because the penetration of gamma ray photons increases with increase in incident gamma ray energy, so the backscattered radiation has to propagate through a large thickness and the flux of multiply backscattered photons having energy equal to the singly backscattered photons reduces (Table 1). Monte Carlo calculations [8] (Fig.3, dotted lines) support the present experimental results.

The parameters called the number $\left(\mathrm{A}_{N}\right)$ and energy $\left(\mathrm{A}_{\mathrm{E}}\right)$ albedos [9] give the effect of multiply backscattered radiations on the original signal (incident photon flux) and characterize the reflection probability of a material for gamma photon flux. 
Sabharwal, A.D. Singh, B.

Sandhu, B.S.

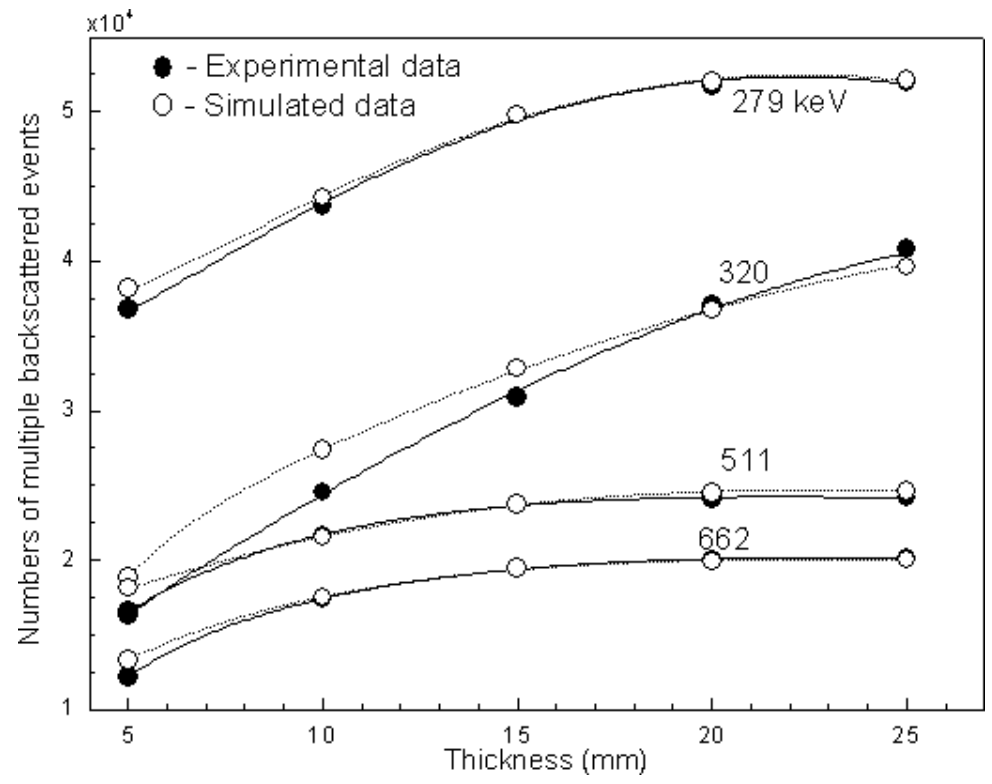

Figure 3: The plots of numbers of multiply backscattered events (experimentally measured as solid-symbol and Monte Carlo simulated as hollow-symbol) as function of copper target thickness for 279-, 320-, $511-$, and $662 \mathrm{keV}$ incident gamma photons

The number albedo [9] is the ratio of total number of reflected photons to the total number of incident photons. The energy albedo refers to the fraction of incident energy that escapes the target. In a similar way, the dose albedo [9] is defined as energy albedo weighted in proportion to the response of the scintillation detector to different photon energies. These albedos are deduced from the data of present experiments for various incident gamma photon energies. The measured number and energy albedos (Table 2) show an increase with increasing target thickness and saturates at the same thickness for which the numbers of multiply backscattered events saturate. The dose albedo does not differ significantly from the energy albedo under present experimental conditions. This behaviour of measured albedos is in agreement with the experimental work of Bulatov and Garusov [3] and with results of Monte Carlo calculations by Perkins [10].

In Compton profile and cross-section measurements these multiply backscattered photons acts as background noise because only the singly backscattered photons are desired. The ratio of number of singly-scattered events to number of multiplybackscattered events (or signal- to-noise ratio) is found to be increasing with increase in target thickness, and its higher value can be achieved by using a thin target and 
Table 2: Experimentally measured values of number and energy albedos for targets of copper at 279, 320, 511 and $662 \mathrm{keV}$ gamma photons under broad beam geometry.

\begin{tabular}{lcccccccc}
\hline $\begin{array}{l}\text { Target } \\
\text { thickness } \\
(\mathrm{mm})\end{array}$ & \multicolumn{2}{c}{$279 \mathrm{keV}$} & \multicolumn{2}{c}{$320 \mathrm{keV}$} & \multicolumn{2}{c}{$511 \mathrm{keV}$} & \multicolumn{2}{c}{$662 \mathrm{keV}$} \\
\cline { 2 - 9 } & $\mathrm{A}_{\mathrm{N}}$ & $\mathrm{A}_{\mathrm{E}}$ & $\mathrm{A}_{\mathrm{N}}$ & $\mathrm{A}_{\mathrm{E}}$ & $\mathrm{A}_{\mathrm{N}}$ & $\mathrm{A}_{\mathrm{E}}$ & $\mathrm{A}_{\mathrm{N}}$ & $\mathrm{A}_{\mathrm{E}}$ \\
\hline 5 & 0.460 & 0.204 & 0.459 & 0.204 & 0.479 & 0.160 & 0.462 & 0.129 \\
10 & 0.462 & 0.204 & 0.464 & 0.206 & 0.480 & 0.160 & 0.468 & 0.130 \\
15 & 0.464 & 0.205 & 0.466 & 0.208 & 0.481 & 0.160 & 0.473 & 0.132 \\
20 & 0.465 & 0.206 & 0.467 & 0.208 & 0.481 & 0.160 & 0.473 & 0.132 \\
25 & 0.465 & 0.206 & 0.467 & 0.208 & 0.481 & 0.160 & 0.473 & 0.132 \\
\hline
\end{tabular}

Energy Dependence of Parameters Characterizing Multiply Backscattering of Gamma Photons

narrow beam collimation. The signal-to-noise ratio is found to be decreasing with increase in incident gamma photon energy.

\section{CONCLUSIONS}

The present measurements provide an independent study of energy dependence of parameters characterizing multiple backscattering of gamma photons. The intensity of multiply backscattered events increases with an increase in target thickness and saturates beyond a particular value, called the saturation thickness. The saturation thickness is found to be decreasing with increase in incident gamma photon energy. The number and energy albedos increase with an increase in target thickness and saturates at the same thickness at which the numbers of multiply backscattered photons saturate. The dose albedo does not differ significantly from the energy albedo for selected incident gamma photon energies. The signal-to-noise ratio is found to be decreasing with an increase in target thickness. Monte Carlo simulations support the results of present experiments. It is further required to carry out measurements using HPGe detector that provides more faithful reproduction of the shape of distribution of the photons originating from the interactions of incident photons with the target. There is also a need to simulate the experiment with the Monte Carlo package including the polarization effects and contributions from moving electrons on multiply backscattered intensity for better understanding of the backscattering of gamma rays.

\section{REFERENCES}

[1] E. Hayward and J.H. Hubbell, J. Appl. Phys., 25, 506 (1954). http://dx.doi.org/10.1063/1.1721671

[2] D.B. Pozdneyev, J. Nucl. Energy, 21, 197 (1967). http://dx.doi.org/10.1016/0022-3107(67)90129-3 
[3] B.P. Bulatov and E.A. Garusov, J. Nucl. Energy, A 11, 159 (1960).

[4] T. Hyodo, Nucl. Sci. \& Engg., 12, 178 (1962).

[5] E. Hayward and J.H. Hubbell, Phys. Rev., 93, 955 (1954). http://dx.doi.org/10.1103/PhysRev.93.955

[6] A. D. Sabharwal, M. Singh, B. Singh and B.S. Sandhu, App. Rad. \& Isot., 66, 1467 (2008). http://dx.doi.org/10.1016/j.apradiso.2008.03.006

[7] A. D. Sabharwal, B. Singh and B.S. Sandhu, Nucl. Instr. \& Meth., B 267, 151 (2009).

[8] G.E.W. Bauer and P. Pattison, Compton scattering experiments at the HMI (Hahn Meitner institute fur kernforschung, Berlin). HMI-B 364 (1981).

[9] A. D. Sabharwal, B. Singh and B.S. Sandhu, Rad. Measurements., 44, 411 (2009). http://dx.doi.org/10.1016/j.radmeas.2009.06.010

[10] J.F. Perkins, J. Appl. Phys., 26, 1372 (1955). http://dx.doi.org/10.1063/1.1722065 